\title{
Effect of Gamma-Ray Energy on Image Quality in Passive Gamma Emission Tomography of Spent Nuclear Fuel
}

\section{Bélanger-Champagne, Camille}

2019-01

Bélanger-Champagne , C, Peura, P, Eerola , P , Honkamaa , T, White , T, Mayorov , M \& Dendooven , P 2019 , ' Effect of Gamma-Ray Energy on Image Quality in Passive Gamma Emission Tomography of Spent Nuclear Fuel ' , IEEE Transactions on Nuclear Science, vol. 66 , no. 1 , pp. 487-496 . https://doi.org/10.1109/TNS.2018.2881138

http://hdl.handle.net/10138/330166

https://doi.org/10.1109/TNS.2018.2881138

acceptedVersion

Downloaded from Helda, University of Helsinki institutional repository.

This is an electronic reprint of the original article.

This reprint may differ from the original in pagination and typographic detail.

Please cite the original version. 


\title{
Effect of Gamma-Ray Energy on Image Quality in Passive Gamma Emission Tomography of Spent Nuclear Fuel
}

\author{
Camille Bélanger-Champagne*, Pauli Peura*§, Paula Eerola*, Tapani Honkamaa ${ }^{\dagger}$, Timothy White ${ }^{\ddagger}$, \\ Mikhail Mayorov $\ddagger$ and Peter Dendooven* \\ *Helsinki Institute of Physics, P.O. Box 64, FI-00014 University of Helsinki, Finland \\ ${ }^{\dagger}$ Radiation and Nuclear Safety Authority - STUK, 00881 Helsinki, Finland \\ ${ }^{\ddagger}$ International Atomic Energy Agency, P.O. Box 100, 1400 Vienna, Austria \\ ${ }_{\S}$ presently at the International Atomic Energy Agency
}

\begin{abstract}
Gamma-ray images of VVER-440 and SVEA-96 spent nuclear fuel assemblies were reconstructed using the filtered back-projection algorithm from measurements with a passive gamma emission tomography prototype instrument at Finnish nuclear power plants. Image quality evaluation criteria based on line profiles through the reconstructed image are used to evaluate image quality for spent fuel assemblies with different cooling times, and thus different mixtures of gamma-ray emitting isotopes. Image characteristics at the locations of water channels and central fuel pins are compared in 2 gamma-ray energy windows, 600-700 $\mathrm{keV}$, and $>700 \mathrm{keV}$, for cooling times up to 10 years for SVEA-96 fuel and 24.5 years for VVER-440 fuel. For SVEA-96 fuel, images in the $>700 \mathrm{keV}$ gamma-ray energy window present better water-to-fuel contrast for all investigated cooling times. For VVER-440, images in the $>700 \mathrm{keV}$ gammaray energy window have higher water-to-fuel contrast up to and including a cooling time of 18.5 years, whereas the water-tofuel contrast of the images taken in the two gamma-ray energy windows are equivalent for a cooling time of 24.5 years. Images reconstructed from higher energy gamma rays such as those in the $>700 \mathrm{keV}$ present better water-to-fuel contrast in fuel cooled for up to 20 years and thus have the most potential for missing fuel pin detection.
\end{abstract}

Index Terms-spent nuclear fuel, gamma-ray tomography, safeguards.

\section{INTRODUCTION}

All signatory countries of the Nuclear Non-Proliferation Treaty have to implement a thorough system of surveillance of their nuclear material. Monitoring is conducted under the IAEA's safeguards system [1]. Spent nuclear fuel (SNF) from nuclear power plants (NPPs) is one of the principal targets of safeguards activities [2]. Most of it is currently stored in wet or dry interim storage at NPPs or dedicated storage facilities.

Passive gamma emission tomography (PGET) has been proposed as a method to provide a cross-sectional image of spent fuel assemblies (SFAs) in a non-destructive assay [3], [4], [5]. The method relies on the strong emission of gamma rays by SFAs, especially by their cesium and europium content. In the image, individual fuel pins can potentially be identified for pinlevel defect testing. Use of gamma emission tomography for partial defect testing in the context of a final deep underground repository was previously investigated under the Finnish IAEA Member State Support Program task FIN A98 [3] and then as a

\begin{tabular}{|l|l|l|l|}
\hline Isotope & $\begin{array}{l}\text { Half-life } \\
\text { [years] }\end{array}$ & $\begin{array}{l}\text { Gamma ray } \\
\text { energy [keV] }\end{array}$ & $\begin{array}{l}\text { Branching } \\
\text { ratio [\%] }\end{array}$ \\
\hline${ }^{134} \mathrm{Cs}$ & 2.07 & 604.7 & 97.6 \\
& & 795.8 & 85.4 \\
\hline${ }^{137} \mathrm{Cs}$ & 30.08 & 661.6 & 85.1 \\
\hline${ }^{144} \mathrm{Ce}-{ }^{144} \mathrm{Pr}$ & 0.78 & 1489.2 & 0.3 \\
& & 2185.6 & 0.7 \\
\hline \multirow{2}{*}{${ }^{154} \mathrm{Eu}$} & 8.60 & 996.3 & 10.3 \\
& & 1004.8 & 17.4 \\
\hline
\end{tabular}

TABLE I: Most important gamma-ray emissions from fission products in medium- and long-cooled spent nuclear fuel.[11], [12], [13], [14]

joint task JNT A1201 of the Finnish, Swedish and Hungarian support programs [6]. Construction of a prototype of a full PGET system began in 2004 and four test campaigns were conducted between 2009 and 2014 under task JNT 1510. A full description of the first PGET prototype instrument and results from the tests can be found in [7]. The prototype was upgraded, and new data taking campaigns in Sweden and Finland were conducted in early 2017. PGET was approved as an inspection instrument by the IAEA in December 2017.

Fission products are the main sources of gamma rays in SNF [8], [9]. Among the gamma-ray-emitting fission products, only those with high activity when coming out of the reactor and long half-lives have the potential to be observed in fuel with a cooling time longer than 10 years [10]. The most relevant isotopes are ${ }^{134} \mathrm{Cs},{ }^{137} \mathrm{Cs},{ }^{144} \mathrm{Ce}-{ }^{144} \mathrm{Pr}$ and ${ }^{154} \mathrm{Eu}$. A list of the most relevant gamma-ray emissions from these isotopes, along with their branching ratios, is shown in Table I.

One of the main challenges of the PGET method is the high self-shielding power of spent fuel. The main material in SFAs, $\mathrm{UO}_{2}$, has a mass-attenuation coefficient of $0.1207 \mathrm{~cm}^{2} \mathrm{~g}^{-1}$ for the $661.6 \mathrm{keV}$ gamma-ray emission from ${ }^{137} \mathrm{Cs}$. This coefficient decreases with increasing gamma-ray energy, reaching $0.0628 \mathrm{~cm}^{2} \mathrm{~g}^{-1}$ for the ${ }^{154} \mathrm{Eu}$ gamma-ray emission at $1274.4 \mathrm{keV}$ [15]. Thus, despite the shorter halflife of higher-energy gamma-ray emitters such as ${ }^{154} \mathrm{Eu}$, use of higher energy gamma rays in image reconstruction has the potential to result in better quality images, especially at the 
centre of the SFAs. This consideration is especially important when looking at large, densely packed fuel, in particular for VVER-1000 and VVER-1200 fuel where 312 fuel pins are arranged in a hexagonal geometry with a total width of only $235 \mathrm{~mm}$ [16]. PGET performance tests on VVER-1000 are planned under task CZ A 1566 of the Czech IAEA Member State support program [17].

In this article, we use data collected by the PGET prototype instrument at the Loviisa and Olkiluoto nuclear power plants in 2017 to reconstruct fuel images using gamma rays from different energy windows and evaluate the quality of the images in the centre of the fuel assembly as a function of the SFA's cooling time. There is no established method for missing fuel pin determination in PGET images, but as it is a safeguards activity, proposed methodology typically relies on relative differences in intensity within the fuel image [4], [7], [18], [19]. Therefore, higher contrast in the image between fuel pins and other areas is beneficial, no matter what specific missing fuel pin determination method is used. In line with safeguards purposes, this study uses a well-established image reconstruction method, filtered backprojection, that uses no prior information about the fuel assembly in the image reconstruction.

\section{Materials AND Methods}

\section{A. The upgraded PGET instrument}

The PGET prototype instrument underwent an upgrade during the period 2015-2016, in which the detectors and electronics were replaced. The new electronics were also accompanied by a new software system. The upgrade was performed by GBS Elektronik GmbH, Germany, in collaboration with the IAEA [18]. An overall description of the upgraded prototype is given in this section.

1) System overview: The PGET prototype is a multidetector instrument designed for gamma imaging of nuclear fuel. An exploded view of the PGET prototype is shown in Figure 1. It is made up of two detector heads, each containing 87 cadmium-zinc-telluride (CZT) detectors, slotted into 8 back-end board modules that can each hold a row of 13 individual detectors, with a detector pitch of $4 \mathrm{~mm}$. The CZT detector crystals have a quasi-hemispherical electric field design and their dimensions are $2 \times 4.8 \times 4.8 \mathrm{~mm}^{3}$. The energy resolution of each detector is better than $3 \%$ FWHM at $661.6 \mathrm{keV}$. The detectors are located behind a $10-\mathrm{cm}$-thick tungsten parallel-slit collimator. The collimator slit pitch is $4 \mathrm{~mm}$ and each opening is $1.5 \mathrm{~mm}$ wide. The collimator opening is angled so that the opening is $70 \mathrm{~mm}$ tall at the front of the collimator, decreasing to $5 \mathrm{~mm}$ at the back. The CZT detectors are located behind the collimator, and are oriented so that a $2 \times 4.8 \mathrm{~mm}^{2}$ side covers the $1.5 \times 5 \mathrm{~mm}^{2}$ opening. On all sides except in front of the collimator, the detector head is shielded with at least $20 \mathrm{~mm}$ of tungsten.

The two detector heads are attached to a rotating plate that can turn $360^{\circ}$. The rotation of the plate is driven by a motor that can be operated in continuous mode or in stepping mode. The two heads are mounted on the plate $180^{\circ}$ apart, with a $2 \mathrm{~mm}$ offset such that data from the two detector heads can be

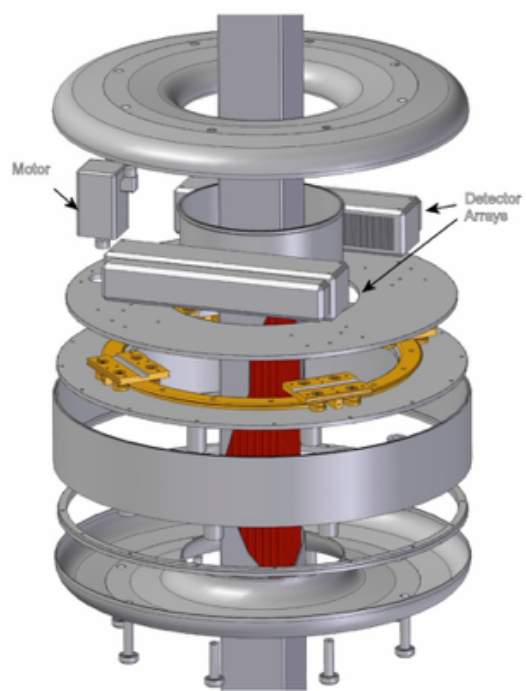

Fig. 1: Exploded view of the PGET prototype including the water tight enclosure, rotating plate, motor and detector heads. [7]

interleaved, resulting in an effective detector spacing of $2 \mathrm{~mm}$. Two boron tube neutron detectors are mounted on opposite sides of the rotating plate, between the CZT detector banks. They provide a gross neutron count measurement which will not be discussed in this article.

The rotating plate, CZT detector heads, neutron detectors and all associated electronics are contained in a toroid-shaped water-tight enclosure shaped from $3 \mathrm{~mm}$ thick stainless-steel sheets. Double o-ring seals are used to insure water tightness for all the joints of the toroidal enclosure.

2) Data acquisition and electronics: The signal collected by each CZT detector is processed by a preamplifier and a shaping amplifier before being sent through a discriminator. The discriminator has four thresholds that can be adjusted individually for each detector. The data acquisition system outputs the number of counts in a user-specified time interval in four user-specified detected gamma-ray energy windows. The first three windows include the counts between pairs of consecutive energy thresholds while the fourth window contains all counts above the highest energy threshold. Ethernet cables supply power to the electronics module and transmit data out of the detector heads. The discriminator threshold for each channel is calibrated in the laboratory using a ${ }^{137} \mathrm{Cs}$ source, and can be recalibrated on-site for each data taking campaign using a spent nuclear fuel assembly as the calibration source. A 1point energy calibration is done using the photopeak from the $661.6 \mathrm{keV}{ }^{137} \mathrm{Cs}$ gamma ray.

\section{B. Characteristics of Finnish spent nuclear fuel}

Two nuclear power plants operate currently in Finland, with a total of four reactors. The Loviisa Nuclear Power Plant is operated by Fortum Oy and consists of two VVER-440/213 pressurized water reactors. The Olkiluoto Nuclear Power Plant is operated by Teollisuuden Voima Oyj and consists of two boiling water reactors. A third reactor, of EPR type, is under construction at the Olkiluoto Nuclear Power Plant. 


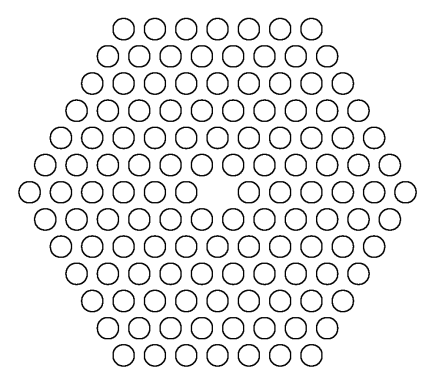

(a) VVER-440

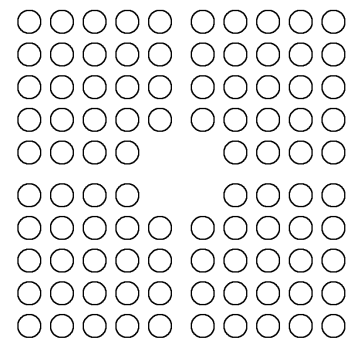

(b) SVEA-96

Fig. 2: Simplified schematic representation, to scale, of (a) VVER-440 and (b) SVEA-96 fuel assemblies.

Two data collection campaigns were performed in Finland with the PGET instrument in early 2017. At the Loviisa NPP, 26 items, 22 of which were spent fuel assemblies of type VVER-440, were measured in the spent fuel storage pool. VVER-440 fuel consists of 126 fuel pins in a hexagonal shape $145 \mathrm{~mm}$ in width, with a single water channel in the centre. Fuel pellets have a diameter of $7.57 \mathrm{~mm}$ and the pin-to-pin pitch is $12.2 \mathrm{~mm}$ [16]. A simplified schematic representation of the fuel pin geometry is shown in Figure 2a.

At the Olkiluoto power plant, 18 items were measured in the reactor-2 fuel pool. These included spent fuel assemblies of types GE 12, SVEA-96, SVEA-100 and Atrium 10, as well as a pin container, a fuel assembly frame where pins, previously removed from different fuel assemblies, are kept for storage. Only images from SVEA-96 SFAs will be shown in this paper. A simplified schematic representation of the SVEA-96 fuel pin geometry is shown in Figure $2 \mathrm{~b}$. This fuel assembly geometry is composed of 96 fuel pins inside a $138.6 \times 138.6 \mathrm{~mm}^{2}$ square, divided into 4 quadrants separated by a cross-shaped open central channel. Fuel pellets have a diameter of $8.48 \mathrm{~mm}$ and a pin-to-pin pitch of $12.8 \mathrm{~mm}$ [20].

\section{Data analysis}

All data acquisition runs used in this paper were performed with the motor operating in continuous mode. The data acquisition software aggregates the data over $1^{\circ}$ so that 360 data projections are measured for each assembly. The measurement time for each projection was $800 \mathrm{~ms}$ for a total measurement time of approximately 5 minutes.

The data collected by the PGET prototype is assembled in a sinogram for each energy window. In this paper, data from 2 energy windows is used for image reconstruction: 600$700 \mathrm{keV}$ and $>700 \mathrm{keV}$. Examples of sinograms (after preprocessing corrections described in Section II-C1) for VVER-440 and SVEA-96 fuel are shown in Figure 3.

1) Data preprocessing and corrections: Data preprocessing is necessary to correct for non-uniformity of behaviour among the CZT detectors in the PGET prototype. SFAs are more intense gamma-ray sources than can typically be found in laboratories. Thus, the preprocessing corrections have to be performed as data-driven self-corrections.

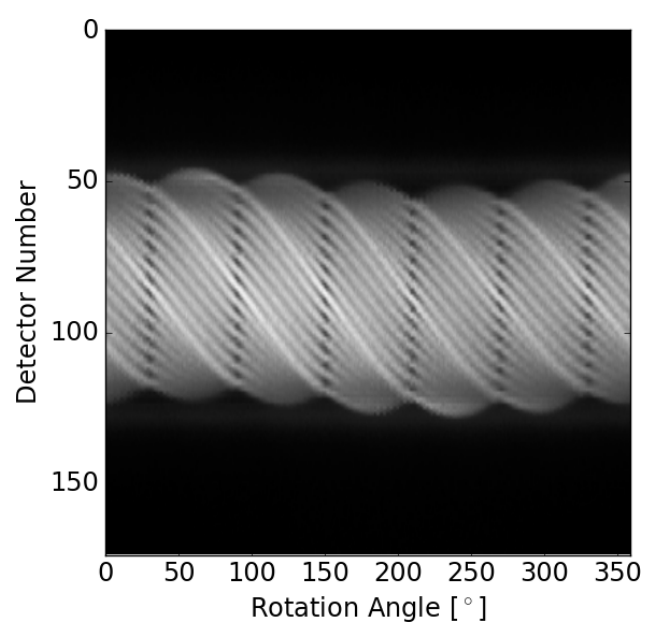

(a) VVER-440

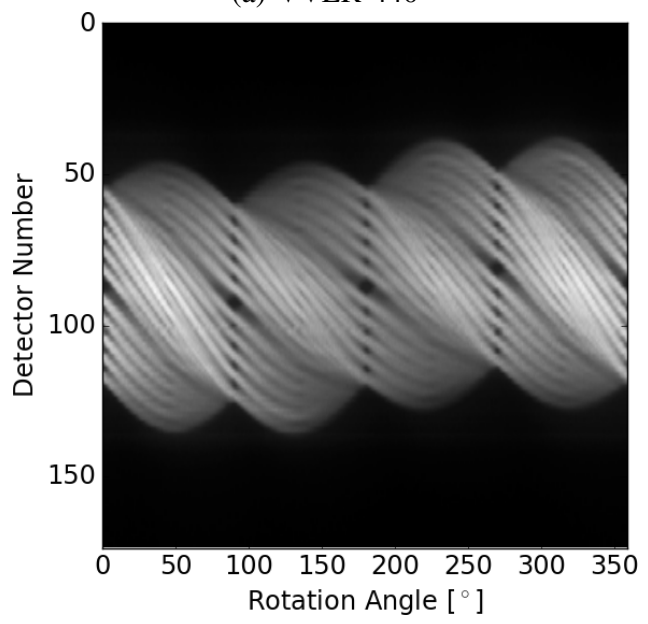

(b) SVEA-96

Fig. 3: Sinogram examples for (a) VVER-440 and (b) SVEA96 SFAs, after data corrections, in the $600-700 \mathrm{keV}$ energy window. The data from both detector heads has been interleaved so that sinograms have an effective detector spacing of $2 \mathrm{~mm}$. The respective 6 -fold and 4-fold symmetry of the fuel assemblies is visible in the lobe-like structure of the sinograms.

First, the data is corrected for deadtime. The deadtime fraction is calculated by the data acquisition system as the fraction of time that the input voltage to the discriminator of a given energy window is above the threshold value. It is stored in the output datastream, individually for each detector threshold and each projection. The number of counts above threshold $n$ is corrected up from the raw count value $n_{r}$ by the deadtime value $d$ so that $n=\frac{n_{r}}{1-d}$.

After the deadtime correction, the individual detector performance is assessed. Some detectors in the PGET prototype showed poor performance in the high rate environment $\left(>10^{5} \mathrm{cps}\right)$ caused by the presence of a SFA. This is understood to be an effect of detector polarization [21]. Above some input gamma-ray flux, the output signal of the detector is constant at a too low or too high value, instead of varying proportionally with the input flux. This can be the case for all angles in a measurement, or for some angular ranges in the measurement, depending mainly on the position of the 


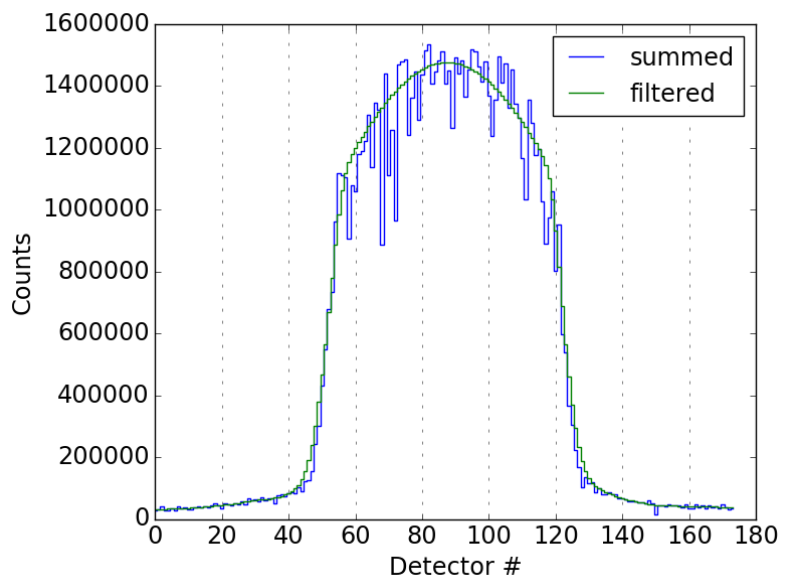

Fig. 4: Example of a summed sinogram, in blue, where the data of the sinogram is projected onto the detector axis. The composite Gaussian filtered summed sinogram used in the data correction is shown in green. The data comes from an assembly of type VVER-440 in the 600-700 keV energy window and detectors \#56, 74, 97, 110 and 118 were interpolated before the sinogram was summed.

fuel within the measurement area. Count values for detectors that are completely inoperable or that display extremely poor response in a given measurement were replaced, for each projection in the sinogram, by the average of the counts of the neighbouring detectors. Because of the nature of this correction, two neighbouring channels cannot be interpolated in this way, so it is used only for the worst-performing detectors. The choice of which detectors to interpolate before image reconstruction was done iteratively for each measurement. The non-uniformity correction, described in the next paragraphs, is performed, and an image is reconstructed. If ring artefacts are visible in the image, the detector causing each ring artefact is selected for interpolation. The process is repeated until no ring artefacts remain in the image.

After all poorly-preforming detectors are interpolated, a global correction for non-uniformity in response among the detectors in the PGET prototype is performed as an in-situ, data-driven, per measurement correction. This correction relies on the fact that, because the detector banks rotate $360^{\circ}$, the projection of the sinogram on the detector axis should result in a smoothly varying curve, as each detector, in total, sees a count rate very similar to that of its neighbours. A typical summed sinogram for VVER fuel is shown in Figure 4.

The summed sinogram is smoothed using Gaussian filtering. For each sinogram, three filtered curves are calculated with Gaussian kernel $\sigma$ values of 2, 5 and 35 for VVER-440 fuel and 2, 7 and 40 for SVEA-96 fuel. A composite filtered summed sinogram is built by combining the filtered curves for the background ( $\sigma=2)$, fuel-edge ( $\sigma=5$ or 7) and fuel $(\sigma=35$ or 40$)$ regions. The background regions correspond to the detectors on each side of the instrument that never have a direct line-of-sight to the fuel, the fuel region is made up of the central or near-central block of detectors that have the fuel in their line-of-sight at all or nearly all measurement angles, and the steeply-sloped fuel-edge regions are made up of the remaining detectors on each side of the fuel region. Detectors are assigned to a region for each measurement based on the fuel position in the instrument during the measurement. The composite curve is filtered again using a Gaussian filter with $\sigma=2$ to ensure that transitions are smooth between regions of the composite. An example of the filtered summed sinogram is shown in Figure 4. The number of counts for each detector in each projection of the sinogram is corrected by the ratio of the filtered to the non-filtered summed sinograms.

2) Image reconstruction: Image reconstruction from the sinogram is done using filtered backprojection on the corrected sinogram data, as implemented in the scikit-image Python module [22], using a Shepp-Logan filter. Due to the lattice structure of the object being imaged, and the high gamma-ray attenuation of $\mathrm{UO}_{2}$, water channels and missing pin locations are not reconstructed as zero-emission pixels in the image. Instead, faint "ghost" pins are visible in the image at the known water locations. The absence of an attenuation correction in the image reconstruction also results in a pronounced "cupping" effect in the image [23]. Extremely few SFAs with missing pins are available at the Loviisa and Olkiluoto NPPs, so the water locations at the centre of the VVER-440 and SVEA-96 SFAs were used as surrogate locations to estimate the ability to detect missing fuel pins in images reconstructed using different gamma-ray energy windows.

3) Image analysis: The position of the fuel pin rows in the image is determined by performing a peak-finding algorithm on the projection of the whole image onto a line that is perpendicular to two of the parallel sides of the hexagonal fuel. Line profiles are constructed from 2-pixel-wide (4-mmwide) strips chosen in the image at the position of each row of fuel pins. The 2-pixel-wide strip is projected onto a single line to create a profile of the fuel pin emission intensity in this row. Peak finding is used on the resulting profile to find the position of each pin in the row. The sum of the value in the peak bin of the profile and its highest neighbour is assigned to be the intensity of the fuel pin or water location. This intensity is thus calculated over a $2 \times 2$ pixel $^{2}\left(4 \times 4 \mathrm{~mm}^{2}\right)$ area, which was chosen such that it is fully enclosed within the cross-sectional area of a fuel pin.

a) VVER-440 image contrast: The water-to-fuel contrast parameter $D_{W, V V E R}$ is defined to be the mean difference between water and fuel pin intensities in the fuel image. It is given by:

$$
D_{W, V V E R}=\frac{1}{6} \sum_{i=1}^{6}\left(P_{F_{i}}-P_{W}\right),
$$

where $P_{W}$ is the peak intensity at the water location shown in dark blue in Figure 5a while $P_{F_{i}}$ is the fuel pin intensity at one of the six fuel locations of the inner ring of fuel pins, shown in light gray in Figure 5a. For hexagonal-shaped VVER-440 fuel, 3 axes of symmetry exist and thus line profiles can be defined in 3 directions along these axes, going through the water location at the centre of the assembly. Each line profile is used to calculate the contributions of two of the six fuel 
pins to the value of $D_{W, V V E R}$. Larger values of $D_{W, V V E R}$ are desirable, as they signal better contrast between the central water channel and neighbouring fuel pins.

b) SVEA-96 image contrast: The SVEA-96 fuel assemblies have a square shape, so line profiles are constructed along 2 orthogonal directions going through rows of fuel pins. The fuel assembly geometry features a wide cross-shaped gap through the centre, which must be taken into account when designing image quality metrics for this fuel assembly geometry. It is important to avoid comparing the intensity of water or fuel locations along the gap to locations that are completely surrounded by fuel: the uneven spacing of the fuel locations affects the uniformity of the intensity of the fuel pins in the reconstructed image. The water and fuel locations used in the image quality metrics defined for SVEA-96 fuel are shown in Figure 5b, along with the quadrant labelling used. The water-to-fuel contrast parameter $D_{W, S V E A}$ is defined as

$$
D_{W, S V E A}=\frac{1}{8} \sum_{Q=1}^{4}\left(\left(P_{F 1_{Q}}-P_{W_{Q}}\right)+\left(P_{F 2_{Q}}-P_{W_{Q}}\right)\right),
$$

where $P_{W_{Q}}$ is the (dark blue) water location in a given quadrant $Q$ and $P_{F 1}$ and $P_{F 2_{Q}}$ are the two central (light gray) neighbouring fuel locations in the same quadrant, as shown in Figure $5 \mathrm{~b}$. Larger values of $D_{W, S V E A}$ are desirable, as they indicate better contrast between the water channel and neighbouring fuel pins.

c) Significance of the water-to-fuel contrast: We define $D_{F}$, the mean pin intensity of the $\mathrm{N}$ central fuel pins, as:

$$
D_{F}=\frac{1}{N} \sum_{i=1}^{N} P_{F_{i}},
$$

where $P_{F_{i}}$ is the fuel pin intensity at one of $N$ central fuel pin locations in the assembly. There are six such locations in VVER-440 fuel and eight such locations in SVEA-96 fuel, shown in light gray in Figure 5. The uncertainty on $D_{W, V V E R}$, $D_{W, S V E A}$ and $D_{F}$ is $S D_{F}$, the standard deviation of the distribution of central fuel pin intensities. It is indicative of the noise level in the determination of pin intensities in the image at the centre of the SFAs.

To establish whether the difference between the peak intensity at the water channel and fuel pin locations is significant relative to the variation in the observed fuel pin intensities, we define $\sigma_{W F}$, as:

$$
\sigma_{W F}=\frac{D_{W, V V E R}}{S D_{F}} \text { or } \frac{D_{W, S V E A}}{S D_{F}},
$$

according to fuel type. Higher values of $\sigma_{W F}$ indicate a more significant contrast between water and fuel locations in the image. In the analysis framework of the Rose criterion [24], each potential fuel rod location is a "picture element". For an image with less than $10^{3}$ picture elements, that is a SFA geometry with less than $10^{3}$ fuel pin locations, $\sigma_{W F}>3$ is the threshold level to clearly distinguish water locations above the intensity fluctuations of neighbour fuel pins. Images of such contrast quality could form the basis for a missing-fuel-pin idenfication algorithm.

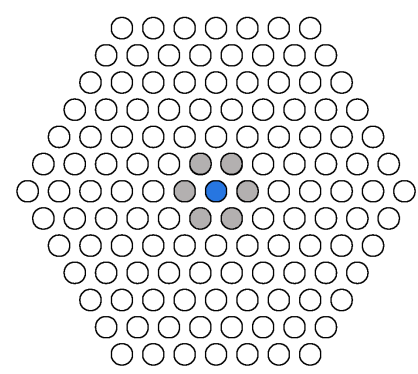

(a) VVER-440

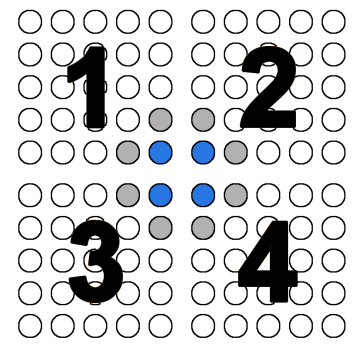

(b) SVEA-96
Fig. 5: Schematic representation of the position of the water locations (dark blue) and central fuel pin locations (light gray) used in the calculation of the intensity difference image quality metric for (a) VVER-440 fuel and (b) SVEA-96 fuel. In SVEA-96 fuel, each location is also labelled by quadrant, with the ordering shown in this figure.

\section{RESULTS}

\section{A. Gamma-ray energy spectra}

Gamma-ray spectra collected for two VVER-440 SFAs with cooling times of $2.5 \mathrm{y}$ and $14.6 \mathrm{y}$ and burnups of $47.5 \mathrm{GWd} / \mathrm{tU}$ and $38.3 \mathrm{GWd} / \mathrm{tU}$, respectively, are shown in Figure 6. The gamma-ray spectrum collected for a SVEA-96 SFA with a 1.9 y cooling time and a $47.0 \mathrm{GWd} / \mathrm{tU}$ burnup is also shown in Figure 6.

These gamma-ray energy spectra show that the sum of the $661.6 \mathrm{keV}$ photopeak of ${ }^{137} \mathrm{Cs}$ and the $604.7 \mathrm{keV}$ photopeak of ${ }^{134} \mathrm{Cs}$ contributes between 12 and $16 \%$ of the counts in the $600-700 \mathrm{keV}$ energy window for the two shorter-cooled assemblies. The short half-life of ${ }^{134} \mathrm{Cs}, 2.06 \mathrm{y}$, means that the contribution from the $604.7 \mathrm{keV}$ photopeak rapidly loses importance with cooling time. In the spectrum from the $14.6 \mathrm{y}$ cooled VVER-440 assembly, the $604.7 \mathrm{keV}$ photopeak from ${ }^{134} \mathrm{Cs}$ is no longer detectable and $50 \%$ of the counts in the $600-700 \mathrm{keV}$ energy window are in the $661.6 \mathrm{keV}$ photopeak of ${ }^{137} \mathrm{Cs}$. The rest of the counts in this energy window are downscattered events from higher energy gamma rays, mainly from ${ }^{154} \mathrm{Eu}$ but also, for the shorter-cooled fuel, from ${ }^{144} \mathrm{Ce}-{ }^{144} \mathrm{Pr}$. As the energy windows used in the analysis are defined for detected gamma-ray energy in each detector, gamma rays that Compton scatter in a CZT detector without depositing all their energy contain correct image information. On the other hand, gamma rays that scatter in the fuel, the water or the collimator and then deposit some or all of their energy in a CZT detector contribute to image noise.

Above $700 \mathrm{keV}$, the main spectrum contribution is the Compton continuum from ${ }^{154} \mathrm{Eu}$ gamma-rays, and, for short-cooled fuel, from ${ }^{144} \mathrm{Ce}-{ }^{144} \mathrm{Pr}$. The photopeak of the $795.8 \mathrm{keV}$ gamma ray from ${ }^{134} \mathrm{Cs}$ is also located within this energy range. The Compton edge associated with this gammaray photopeak falls below the $700 \mathrm{keV}$ energy threshold for this window and mainly below the $600 \mathrm{keV}$ energy threshold of the other measurement window. In the shortest-cooled fuel 


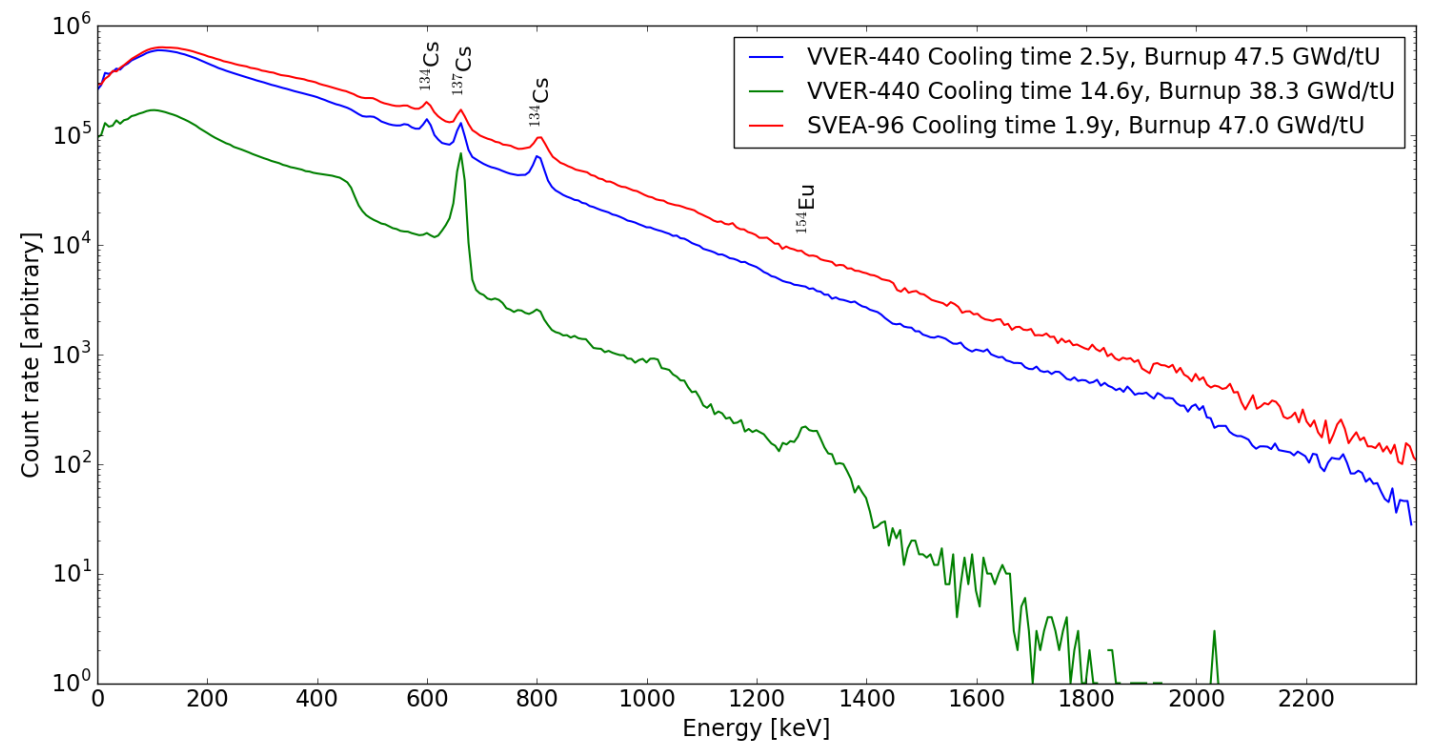

Fig. 6: Sum of the gamma-ray energy spectra collected by 15 individual CZT detectors in the centre of the PGET detector heads for two VVER-440 assemblies (short and medium-cooled, blue and green lines, respectively) and one SVEA-96 assembly (short-cooled, red line).

measured during the 2017 campaigns, as shown in Figure 6, the contribution of the $795.8 \mathrm{keV}$-photopeak to the total counts in the over $700 \mathrm{keV}$ energy window is approximately $4 \%$. It has completely decayed away in the spectrum from the $14.6 \mathrm{y}-$ cooled VVER-440 assembly. The photopeak efficiency of the detector decreases with increasing gamma-ray energy [25], which is why no significant photopeaks are visible for the ${ }^{154} \mathrm{Eu}$ and ${ }^{144} \mathrm{Ce}-{ }^{144} \mathrm{Pr}$ gamma rays.

\section{B. VVER-440 fuel}

For four SFAs with varying cooling times and burnups, reconstructed images in 2 energy windows are shown in Figure 7 and example line profiles though the centre of the assembly are shown in Figure 8 for one of the three line profile directions. Line profiles along the other directions were also constructed and they were used to calculate $D_{W, V V E R}$ and $D_{F}$, but are not shown as they do not present any significant differences relative to the ones shown.

The values of $D_{W, V V E R}, D_{F}, S D_{F}$ and $\sigma_{W F}$, calculated according to the method described in Section II-C3 for these four VVER-440 SFAs, are listed in Table II.

\section{SVEA-96 fuel}

Gamma-ray images and line profiles for the central rows of four SVEA-96 SFAs are shown in Figure 9 and 10 for one of the two line profile directions investigated. Line profiles along the other direction were also constructed but present no significant differences relative to the profiles shown.

The values of $D_{W, S V E A}, D_{F}, S D_{F}$ and $\sigma_{W F}$, calculated according to the method described in Section II-C3 for these four SVEA-96 SFAs, are listed in Table III.

\begin{tabular}{|c|c|c|c|}
\hline \multirow{3}{*}{$\begin{array}{c}\text { Cooling } \\
\text { time } \\
\text { [years] }\end{array}$} & & \multicolumn{2}{|c|}{ Energy window } \\
\cline { 3 - 4 } & & $600-700 \mathrm{keV}$ & $>700 \mathrm{keV}$ \\
\hline \multirow{4}{*}{2.5} & $D_{W, V V E R}$ [a.u.] & $0.16 \times 10^{3}$ & $0.34 \times 10^{3}$ \\
\cline { 2 - 4 } & $D_{F}$ [a.u.] & $1.41 \times 10^{3}$ & $2.07 \times 10^{3}$ \\
\cline { 2 - 4 } & $S D_{F}$ [a.u.] & $0.04 \times 10^{3}$ & $0.05 \times 10^{3}$ \\
\cline { 2 - 4 } & $\sigma_{W F}$ & 4.5 & 6.2 \\
\hline \multirow{4}{*}{13.6} & $D_{W, V V E R}$ [a.u.] & 35 & 16 \\
\cline { 2 - 4 } & $D_{F}$ [a.u.] & 305 & 103 \\
\cline { 2 - 4 } & $S D_{F}$ [a.u.] & 7 & 3 \\
\cline { 2 - 4 } & $\sigma_{W F}$ & 4.9 & 5.3 \\
\hline \multirow{4}{*}{18.5} & $D_{W, V V E R}$ [a.u.] & 24 & 9.9 \\
\cline { 2 - 4 } & $D_{F}$ [a.u.] & 237 & 60.9 \\
\cline { 2 - 4 } & $S D_{F}$ [a.u.] & 5 & 1.5 \\
\cline { 2 - 4 } & $\sigma_{W F}$ & 5.1 & 6.6 \\
\hline \multirow{4}{*}{24.5} & $D_{W, V V E R}$ [a.u.] & 13 & 3.0 \\
\cline { 2 - 4 } & $D_{F}$ [a.u.] & 213 & 40.7 \\
\cline { 2 - 4 } & $S D_{F}$ [a.u.] & 4 & 1.1 \\
\cline { 2 - 4 } & $\sigma_{W F}$ & 2.9 & 2.7 \\
\hline
\end{tabular}

TABLE II: Fuel-to-water image contrast parameter $D_{W, V V E R}$, the fuel pin mean intensity $D_{F}$, the standard deviation of the central fuel pin intensities $S D_{F}$ and the significance of the water-to-fuel contrast $\sigma_{W F}$ of VVER-440 SFAs as a function of the assembly cooling time in two gamma-ray energy windows.

\section{DISCUSSION}

In the images of VVER-440 SFAs, as shown in Table II, $\sigma_{W F}$ is above 3 for both energy windows for all assemblies with a cooling time up to and including $18.5 \mathrm{y}$, indicating that the contrast is sufficient to reliably distinguish the central water location in the assembly from neighbouring fuel pins. For these SFAs, the water-to-fuel contrast is better in the higher energy window, as the value of $\sigma_{W F}$ is at least 0.4- 


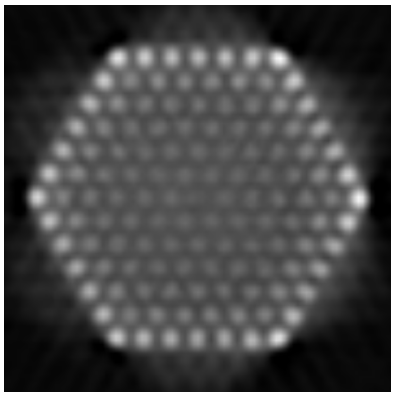

(a) $2.5 \mathrm{y}, 48.6 \mathrm{GWd} / \mathrm{tU}$, $600-700 \mathrm{keV}$

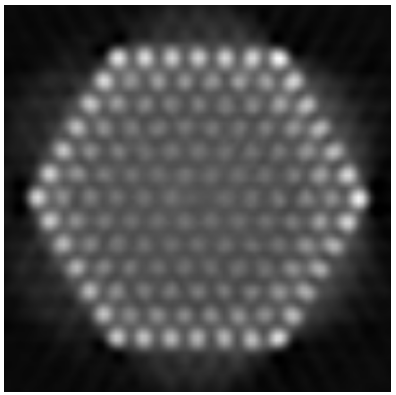

(e) $2.5 \mathrm{y}, 48.6 \mathrm{GWd} / \mathrm{tU}$, $>700 \mathrm{keV}$

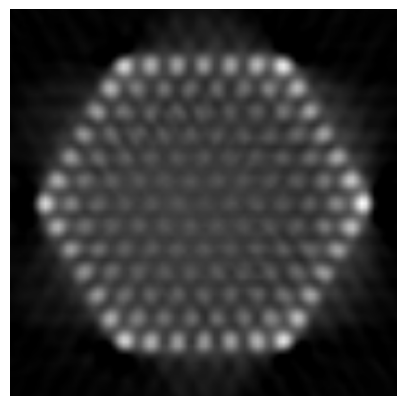

(b) $13.6 \mathrm{y}, 41.7 \mathrm{GWd} / \mathrm{tU}$, $600-700 \mathrm{keV}$

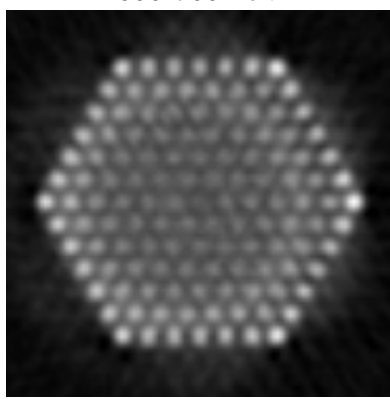

(f) $13.6 \mathrm{y}, 41.7 \mathrm{GWd} / \mathrm{tU}$, $>700 \mathrm{keV}$

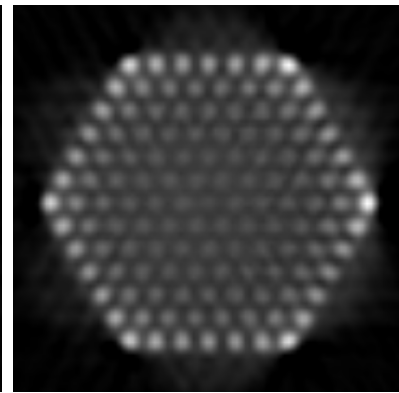

(c) $18.5 \mathrm{y}, 39.3 \mathrm{GWd} / \mathrm{tU}$, $600-700 \mathrm{keV}$

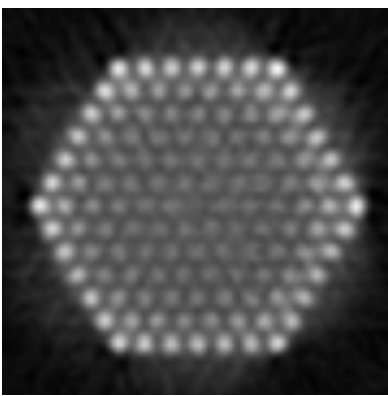

(g) $18.5 \mathrm{y}, 39.3 \mathrm{GWd} / \mathrm{tU}$, $>700 \mathrm{keV}$

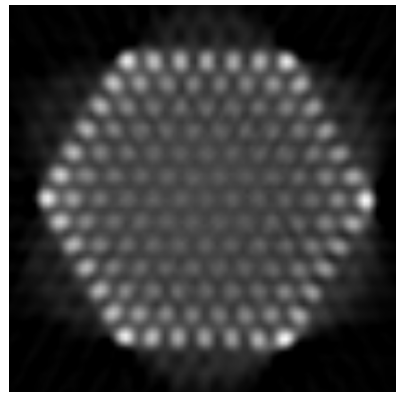

(d) $24.5 \mathrm{y}, 38.2 \mathrm{GWd} / \mathrm{tU}$, $600-700 \mathrm{keV}$

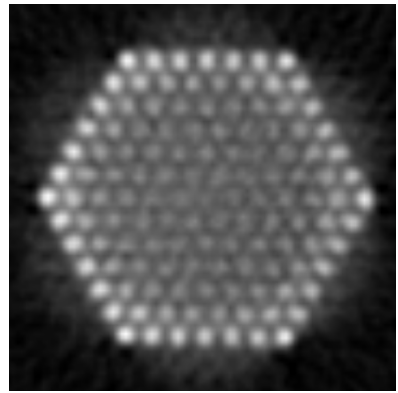

(h) $24.5 \mathrm{y}, 38.2 \mathrm{GWd} / \mathrm{tU}$, $>700 \mathrm{keV}$

Fig. 7: Reconstructed images of VVER-440 SFAs. The cooling time and burnup of the assembly is indicated below each image. Gamma rays from the 600-700 keV energy window are used for (a)-(d) (top row) while gamma rays above $700 \mathrm{keV}$ are used for (e)-(h) (bottom row). Each image is scaled separately to a maximum pixel value of 1 .

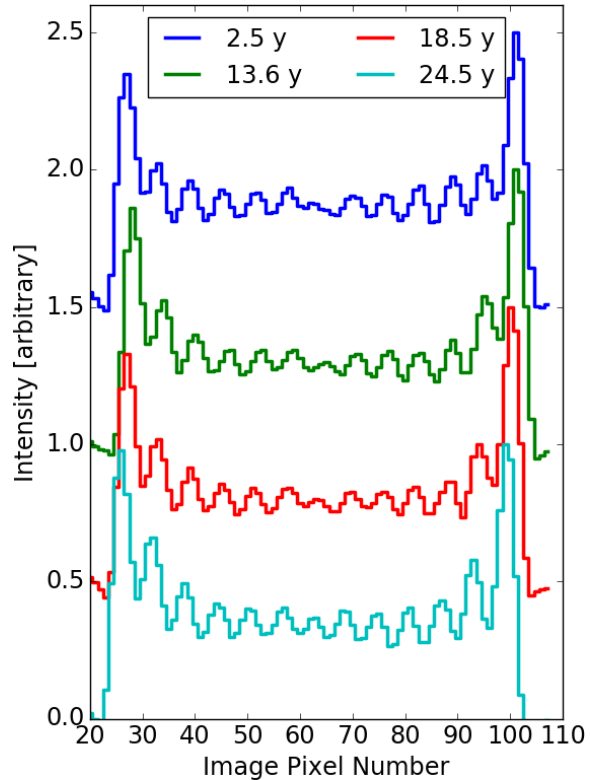

(a) $600-700 \mathrm{keV}$

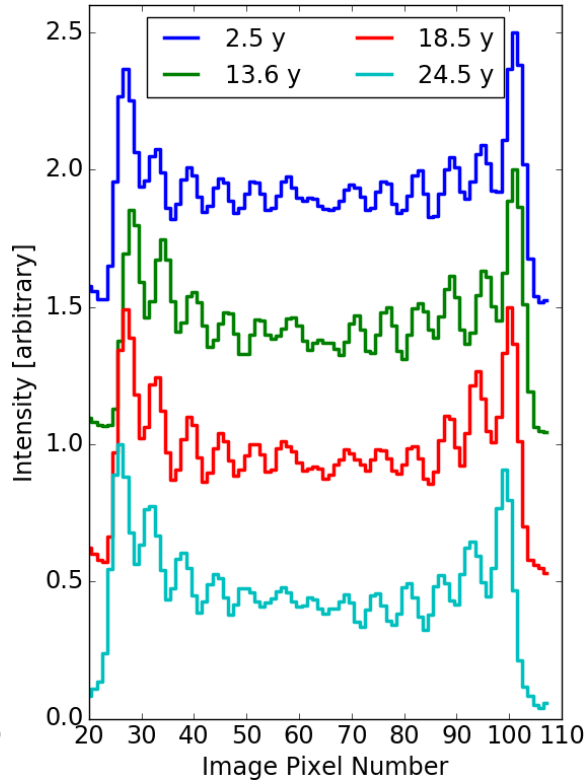

(b) $>700 \mathrm{keV}$

Fig. 8: Line profiles of the central horizontal rows of pins in the images of VVER-440 SFAs shown in Figure 7 for (a) the $600-700 \mathrm{keV}$ and (b) the $>700 \mathrm{keV}$ energy windows. These rows include a water location at the centre, in the image pixel number range 60-65. 


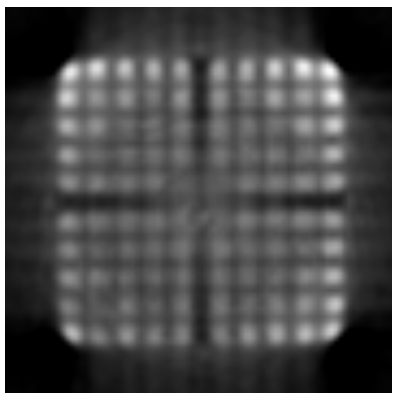

(a) $1.9 \mathrm{y}, 47.0 \mathrm{GWd} / \mathrm{tU}$, $600-700 \mathrm{keV}$

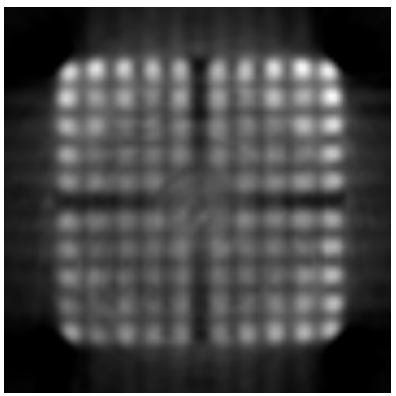

(e) $1.9 \mathrm{y}, 47.0 \mathrm{GWd} / \mathrm{tU}$ $>700 \mathrm{keV}$

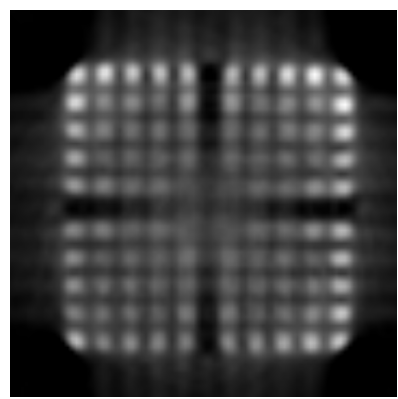

(b) $4.8 \mathrm{y}, 45.2 \mathrm{GWd} / \mathrm{tU}$, $600-700 \mathrm{keV}$

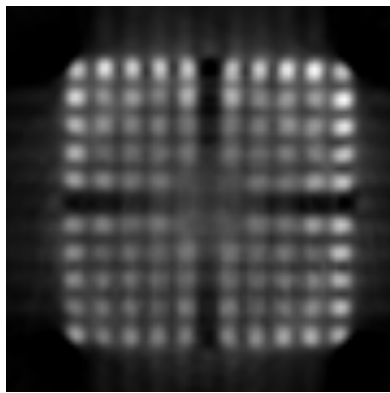

(f) $4.8 \mathrm{y}, 45.2 \mathrm{GWd} / \mathrm{tU}$, $>700 \mathrm{keV}$

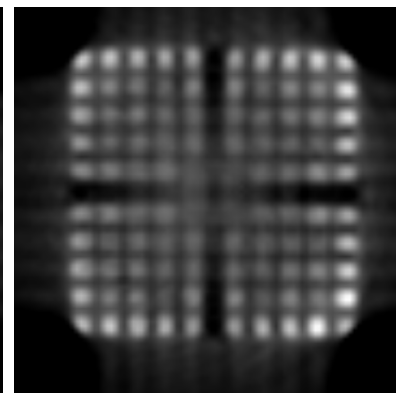

(c) $8.9 \mathrm{y}, 40.7 \mathrm{GWd} / \mathrm{tU}$, $600-700 \mathrm{keV}$

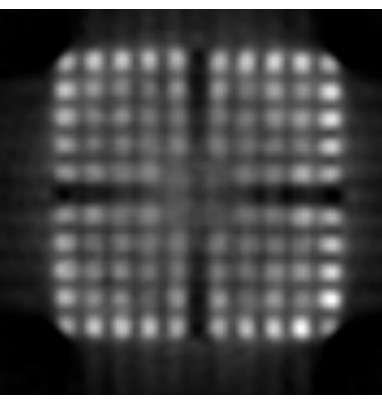

(g) $8.9 \mathrm{y}, 40.7 \mathrm{GWd} / \mathrm{tU}$, $>700 \mathrm{keV}$

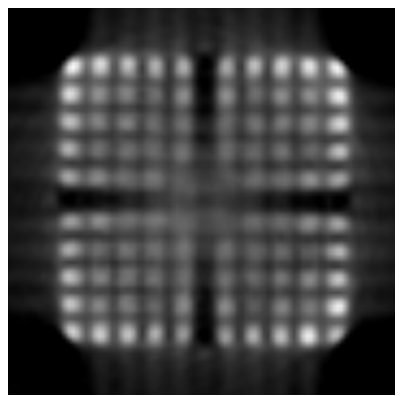

(d) 9.9 y, $50.4 \mathrm{GWd} / \mathrm{tU}$, $600-700 \mathrm{keV}$

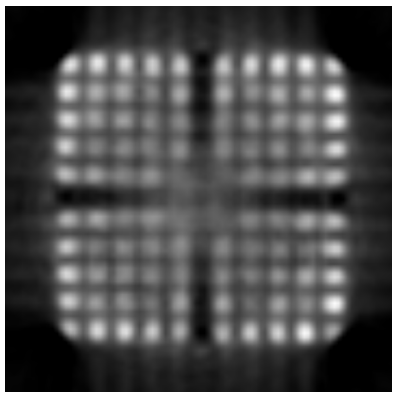

(h) $9.9 \mathrm{y}, 50.4 \mathrm{GWd} / \mathrm{tU}$, $>700 \mathrm{keV}$

Fig. 9: Reconstructed images of SVEA-96 SFAs. The cooling time and burnup of the assembly is indicated below each image. Gamma rays from the 600-700 keV energy window are used for (a)-(d) (top row) while gamma rays above $700 \mathrm{keV}$ are used for (e)-(h) (bottom row). Each image is scaled separately to a maximum pixel value of 1 .

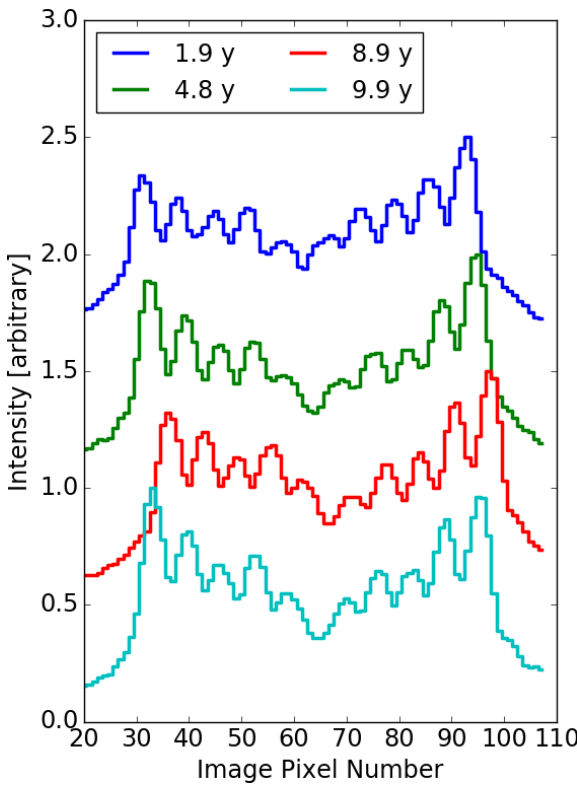

(a) $600-700 \mathrm{keV}$

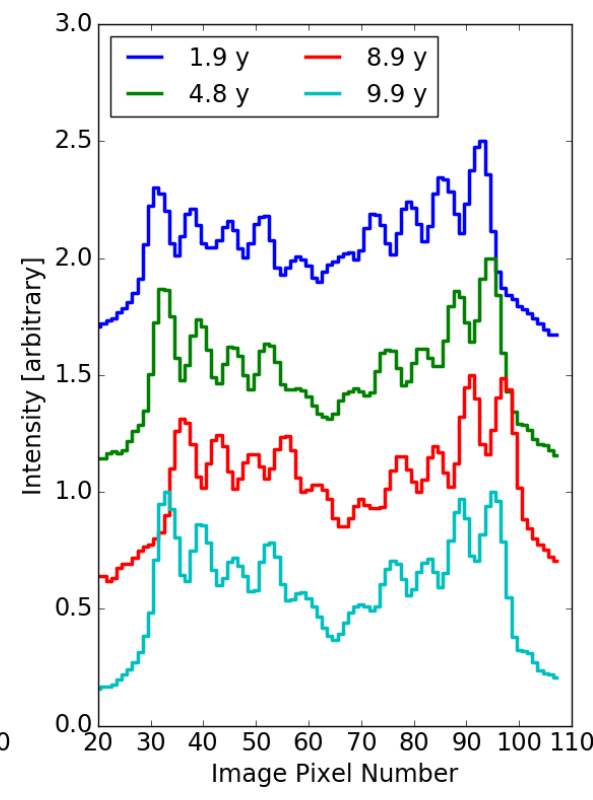

(b) $>700 \mathrm{keV}$

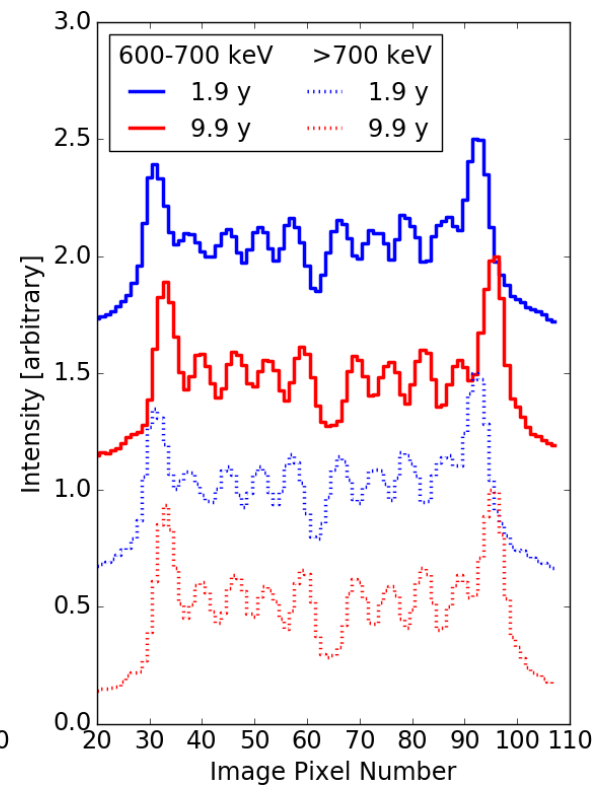

(c) all-fuel rows

Fig. 10: Line profiles of the row of pins immediately above the central cross-shaped gap in the images of SVEA-96 SFAs shown in Figure 9 for (a) the $600-700 \mathrm{keV}$ and (b) the $>700 \mathrm{keV}$ energy window. This row includes two water locations in the image pixel number ranges 55-65 and 65-75. (c) For two of the SFAs, line profiles of the fuel row above the rows plotted in (a) and (b) in the images of Figure 9. These rows do not contain water locations other than the cross-shaped gap, visible in the image pixel number range 60-70. 


\begin{tabular}{|c|c|c|c|}
\hline $\begin{array}{c}\text { Cooling } \\
\text { time } \\
\text { [years] }\end{array}$ & & \multicolumn{2}{|c|}{ Energy window } \\
\cline { 3 - 4 } & & $600-700 \mathrm{keV}$ & $>700 \mathrm{keV}$ \\
\hline \multirow{4}{*}{1.9} & $* D_{W, S V E A}$ [a.u.] & $0.43 \times 10^{3}$ & $0.99 \times 10^{3}$ \\
\cline { 2 - 4 } & $D_{F}$ [a.u.] & $2.42 \times 10^{3}$ & $4.18 \times 10^{3}$ \\
\cline { 2 - 4 } & $S D_{F}$ [a.u.] & $0.06 \times 10^{3}$ & $0.12 \times 10^{3}$ \\
\cline { 2 - 4 } & $\sigma_{W F}$ & 7.4 & 8.1 \\
\hline \multirow{4}{*}{4.8} & $D_{W, S V E A}$ [a.u.] & $0.21 \times 10^{3}$ & $2.6 \times 10^{2}$ \\
\cline { 2 - 4 } & $D_{F}$ [a.u.] & $1.07 \times 10^{3}$ & $9.9 \times 10^{2}$ \\
\cline { 2 - 4 } & $S D_{F}$ [a.u.] & $0.04 \times 10^{3}$ & $0.2 \times 10^{2}$ \\
\cline { 2 - 4 } 8.9 & $\sigma_{W F}$ & 5.3 & 12.6 \\
\hline \multirow{4}{*}{9.9} & $D_{W, S V E A}$ [a.u.] & $1.5 \times 10^{2}$ & $1.0 \times 10^{2}$ \\
\cline { 2 - 4 } & $D_{F}$ [a.u.] & $6.8 \times 10^{2}$ & $3.8 \times 10^{2}$ \\
\cline { 2 - 4 } & $S D_{F}$ [a.u.] & $0.4 \times 10^{2}$ & $0.2 \times 10^{2}$ \\
\cline { 2 - 4 } & $\sigma_{W F}$ & 3.6 & 4.8 \\
\hline \multirow{4}{*}{9.9} & $D_{W, S V E A}$ [a.u.] & $1.6 \times 10^{2}$ & $1.3 \times 10^{2}$ \\
\cline { 2 - 4 } & $D_{F}$ [a.u.] & $8.6 \times 10^{2}$ & $5.5 \times 10^{2}$ \\
\cline { 2 - 4 } & $S D_{F}$ [a.u.] & $0.4 \times 10^{2}$ & $0.3 \times 10^{2}$ \\
\cline { 2 - 4 } & $\sigma_{W F}$ & 4.0 & 5.0 \\
\hline & & & \\
\hline
\end{tabular}

TABLE III: Fuel-to-water image contrast parameter $D_{W, S V E A}$, the fuel pin mean intensity $D_{F}$, the standard deviation of the central fuel pin intensities $S D_{F}$ and the significance of the water-to-fuel contrast $\sigma_{W F}$ of SVEA-96 SFAs as a function of the assembly cooling time in two gamma-ray energy windows.

*Quadrant \#3 is not used for this assembly, see Discussion.

$\sigma$ higher in the $>700 \mathrm{keV}$ window than in the $600-700 \mathrm{keV}$ energy window. For the 24.5 year-cooled assembly, $\sigma_{W F}$ is 2.9 and 2.7 , respectively, in the $600-700 \mathrm{keV}$ window and the $>700 \mathrm{keV}$ window. The higher energy window no longer presents better image contrast. The evolution of the image contrast between the energy windows can also be qualitatively observed in the line profiles shown in Figure 8, where the "ghost" pin in the middle of the row is more visible in the lower energy window and, in each energy window, becomes more similar in intensity to its neighbour fuel pins as cooling time increases.

In the images of SVEA-96 SFAs, $\sigma_{W F}$ is above 3 for both energy windows for all measured assemblies, as shown in Table III, indicating that the contrast in the image is sufficient ot reliably distinguish the water locations near the centre of the assembly from neighbouring fuel pins. Quadrant \#3 was not included in the calculation of $D_{W, S V E A}$ for the $1.9 \mathrm{y}$ cooled assembly. This SFA is the most intense radiation source imaged with the PGET during this campaign and, as such, the degraded detector performance resulted in stronger ring artefacts, some of which remain in the image after detector interpolation and non-uniformity correction. One such artefact is visible near the centre of the assembly image, in the water gap between quadrants \#3 and \#4. This artefact degrades the contrast between water and fuel locations in this area of the image.

The water-to-fuel contrast is better in the higher energy window for all measured SVEA-96 assemblies, as the value of $\sigma_{W F}$ is at least $1-\sigma$ higher in the $>700 \mathrm{keV}$ window than in the 600-700 keV energy window. The contrast in peak intensity between water location and fuel pins can also be qualitatively observed in the line profiles shown in Figure 10. In Figure 10c, neighbour rows without water locations are shown, and we see that the profiles are very different from the rows with water locations.

\section{COnClusions}

Gamma-ray images of SNF were reconstructed using filtered back-projection for VVER-440 and SVEA-96 SFAs measured with a PGET prototype instrument at Finnish NPPs. A water-to-fuel image contrast metric based on image line profiles was used to evaluate the images reconstructed using gamma rays in different energy windows for SFAs with different cooling times. The metric defined in this paper can be used to evaluate the performance of other image reconstruction techniques and can be adapted to evaluate the image quality for different fuel assembly geometries.

Images of SVEA-96 SFAs with cooling times of up to $10 \mathrm{y}$ were reconstructed in both the $600-700 \mathrm{keV}$ and $>700 \mathrm{keV}$ energy windows. In all studied cases, the images present significant contrast $\sigma_{W F}>3$ between water locations and fuel pins near the centre of the assembly. The images reconstructed from gamma rays with energy above $700 \mathrm{keV}$ were of consistently higher contrast than images from the $600-700 \mathrm{keV}$ energy window. For the VVER-440 SFAs imaged in this study, images reconstructed in both the 600-700 keV energy window and the $>700 \mathrm{keV}$ energy window have significant water-tofuel contrast $\sigma_{W F}>3$ at cooling times up to and including $18.5 \mathrm{y}$, and just below 3 for the assembly with a cooling time of $24.5 \mathrm{y}$. For cooling times up to and including $18.5 \mathrm{y}$, the images reconstructed using gamma rays with energy above $700 \mathrm{keV}$ present a more significant water-to-fuel contrast than those from gamma rays in the $600-700 \mathrm{keV}$ energy window. For the assembly with a cooling time of $24.5 \mathrm{y}$, the water-tofuel contrast is equivalent in the two energy windows.

In summary, the lower attenuation of higher-energy gamma rays in spent nuclear fuel results in images with a better water-to-fuel contrast in the centre of the SFAs measured in this study up to a fuel cooling time of 18.5 years, included. This indicates that images reconstructed from the $>700 \mathrm{keV}$ window have a better potential for missing pin detection in fuel cooled for up to approximately 20 years.

\section{REFERENCES}

[1] "Treaty on the non-proliferation of nuclear weapons," United Nations Treaty Series, vol. 729, no. 10485, p. 161, 1970. [Online]. Available: https://www.un.org/disarmament/wmd/nuclear/npt/text

[2] IAEA, Estimation of Global Inventories of Radioactive Waste and Other Radioactive Materials, ser. IAEA Publications, Vienna: International Atomic Energy Agency, 2008, no. IAEA-TECDOC-1591.

[3] F. Lévai, S. Dési, M. Tarvainen, and R. Arlt, Use of high energy gamma emission tomography for partial defect verification of spent fuel assemblies, ser. STUK Reports, 1993, no. STUK-YTO-TR-56.

[4] T. Lundqvist, S. J. Svärd, and A. Håkansson, "SPECT imaging as a tool to prevent proliferation of nuclear weapons," Nuclear Instruments and Methods in Physics Research Section A: Accelerators, Spectrometers, Detectors and Associated Equipment, vol. 580, no. 2, pp. 843 - 847, Oct 2007, imaging 2006. [Online]. Available: http://www.sciencedirect.com/science/article/pii/S0168900207012740 
[5] L. Smith, V. Mozin, E. Miller, N. Deshmukh, R. Wittman, S. Grape, S. Vaccaro, S. Jacobsson-Svard, P. Jansson, T. Honkamaa, T. White, H. Trellue, A. Davour, P. Andersson, and S. Holcombe, A Viability Study of Gamma Emission Tomography for Spent Fuel Verification: JNT 1955 Phase I Technical Report, Pacific Northwest National Laboratory, Richland, WA, USA, 2016, no. PNNL-25995.

[6] F. Lévai, S. Dési, S. Czifrus, S. Feher, M. Tarvainen, T. Honkamaa, J. Saarinen, M. Larsson, A. Rialhe, and R. Arlt, Feasibility of gamma emission tomography for partial defect verification of spent LWR fuel assemblies, ser. STUK Reports, 2002, no. STUK-YTO-TR 189.

[7] T. Honkamaa, F. Levai, A. Turunen, R. Berndt, S. Vaccaro, and P. Schwalbach, "A prototype for passive gamma emission tomography," in Proceedings of the 2014 Symposium on International Safeguards, Vienna, 2014.

[8] P. Cudrnak, "Model analysis of isotopic composition of VVER440 spent nuclear fuel," Journal of Power Technologies, vol. 91, no. 3, pp. 136-139, 2011. [Online]. Available: https://search.proquest.com/docview/1657349199?accountid=11365

[9] I. Günther-Leopold, B. Wernli, Z. Kopajtic, and D. Günther, "Measurement of isotope ratios on transient signals by MC-ICP-MS," Analytical and Bioanalytical Chemistry, vol. 378, no. 2, pp. 241-249, Jan 2004. [Online]. Available: https://doi.org/10.1007/s00216-003-2226-

[10] J. R. Phillips, "Irradiated fuel measurements," in Passive Nondestructive Assay of Nuclear Materials, D. Reilly, N. Ensslin, and J. Hastings Smith, Eds. Los Alamos, LA-UR-90-732: Los Alamos National Laboratory, 1991 , ch. 18 , pp. $529-562$.

[11] A. Sonzogni, "Nuclear Data Sheets for A = 134," Nuclear Data Sheets, vol. 103, no. 1, pp. 1 - 182, Sep 2004. [Online]. Available: http://www.sciencedirect.com/science/article/pii/S0090375204000717

[12] E. Browne and J. Tuli, "Nuclear Data Sheets for A = 137," Nuclear Data Sheets, vol. 108, no. 10, pp. 2173 - 2318, Oct 2007. [Online]. Available: http://www.sciencedirect.com/science/article/pii/S0090375207000804

[13] A. Sonzogni, "Nuclear Data Sheets for A = 144," Nuclear Data Sheets, vol. 93, no. 3, pp. 599 - 762, Jul 2001. [Online]. Available: http://www.sciencedirect.com/science/article/pii/S0090375201900158

[14] C. Reich, "Nuclear Data Sheets for A = 154," Nuclear Data Sheets, vol. 110, no. 10, pp. 2257 - 2532, Oct 2009. [Online]. Available: http://www.sciencedirect.com/science/article/pii/S0090375209000805

[15] J. H. Hubbell, " Photon Cross Sections, Attenuation Coefficients and Energy Absorption Coefficients from $10 \mathrm{keV}$ to $100 \mathrm{GeV}$," NSRDS-NBS 29, 1969.

[16] TVEL JSC, Nuclear fuel for VVER reactors, http://www.tvel.ru/wps/wcm/connect/tvel/tvelsite.eng/resources/ b00eb08047178c38abfbfb39942cc531/brochure_nuclea_eng.pdf (Consulted April 30th 2018)

[17] IAEA Department of Safeguards, "Development and Implementation Support Programme for Nuclear Verification 2018-2019," STR-386, 2018.

[18] M. Mayorov, T. White, A. Lebrun, J. Brutscher, J. Keubler, A. Birnbaum, V. Ivanov, T. Honkamaa, P. Peura and J. Dahlberg, "Gamma emission tomography for the inspection of spent nuclear fuel," in IEEE NSS 2017 Conference Record, Atlanta, Georgia, USA, 2017.

[19] T. White, M. Mayorov, A. Lebrun, P. Peura, T. Honkamaa, J. Dahlberg, J. Keubler, V. Ivanov and A. Turunen, "Application of Passive Gamma Emission Tomography (PGET) for the Verification of Spent Nuclear Fuel," in Proceedings of the INMM 59 th Annual Meeting, Baltimore, Maryland, USA, 2018.

[20] Svensk Kärnbränslehantering $\mathrm{AB}$, "Spent nuclear fuel for disposal in the KBS-3 repository," SKB TR-10-13, 2010.

[21] G. S. Camarda, A. E. Bolotnikov, Y. Cui, A. Hossain, S. A. Awadalla, J. Mackenzie, H. Chen, and R. B. James, "Polarization studies of CdZnTe detectors using synchrotron x-ray radiation," in 2007 IEEE Nuclear Science Symposium Conference Record. IEEE, Oct 2007, pp. 1798-1804. [Online]. Available: http://ieeexplore.ieee.org/document/4436508/

[22] S. van der Walt, J. L. Schönberger, J. Nunez-Iglesias, F. Boulogne, J. D. Warner, N. Yager, E. Gouillart, T. Yu, and the scikit-image contributors, "scikit-image: image processing in Python," PeerJ, vol. 2, p. e453, Jun 2014. [Online]. Available: http://dx.doi.org/10.7717/peerj.453

[23] J. F. Barrett and N. Keat, "Artifacts in CT: Recognition and Avoidance," RadioGraphics, vol. 24, no. 6, p. 1679, 2004.

[24] A. Rose, Vision: Human and Electronic. New York: Plenum Press, 1973.

[25] T. Takahashi and S. Watanabe, "Recent progress in CdTe and CdZnTe detectors," IEEE Trans. Nucl. Sci., vol. 48, no. 4, pp. 950-959, Aug 2001. (c) 2019 IEEE. Personal use of this material is permitted.

Permission from IEEE must be obtained for all other uses, in any current or future media, including reprinting/republishing this material for advertising or promotional purposes, creating new collective works, for resale or redistribution to servers or lists, or reuse of any copyrighted component of this work in other works. 